\title{
Shikonin regulates autophagy via the AMPK/mTOR pathway and reduces apoptosis of human umbilical cord mesenchymal stem cells to improve survival in tissues surrounding brain contusion
}

\author{
XIAOHONG ZHU ${ }^{1,2^{*}}$, LIJIE HUANG ${ }^{1,2^{*}}, \mathrm{KE} \mathrm{WU}^{1,2}$, ZHEZHE SUN $^{1,2}$, KANKAI WANG $^{1,2}$, \\ JUNNAN RU ${ }^{1,2}$, QICHUAN ZHUGE ${ }^{1,2}$ and LINHUI RUAN ${ }^{1,2}$ \\ ${ }^{1}$ Department of Neurosurgery; ${ }^{2}$ Zhejiang Provincial Key Laboratory of Aging and Neurological Disorder Research, \\ The First Affiliated Hospital of Wenzhou Medical University, Wenzhou, Zhejiang 325000, P.R. China
}

Received September 8, 2019; Accepted March 24, 2021

DOI: $10.3892 /$ etm.2021.10910

\begin{abstract}
Shikonin has been reported to regulate autophagy via the AMP-activated protein kinase (AMPK)/mTOR signalling pathway and decrease apoptosis in transplanted human umbilical cord mesenchymal stem cells (HUMSCs). In the present study, HUMSCs were exposed to oxygen glucose deprivation (OGD) in vitro for $12 \mathrm{~h}$, and TUNEL fluorescence staining was used to detect apoptosis. Differences in autophagy and AMPK/mTOR pathway-related protein expression following treatment with shikonin were quantitatively analyzed by western blotting. Green fluorescent protein-labelled stem cells were implanted into traumatic brain injury-model mice and the survival of HUMSCs was observed after 7 days. Shikonin increased the number of cells in brain tissue surrounding the contusion 7 days after transplantation. Furthermore, shikonin treatment decreased apoptosis, increased the expression of autophagy-related proteins, increased phosphorylated AMPK expression and downregulated phosphorylated mTOR expression. In addition, the autophagy inhibitor 3-methyladenine attenuated these effects and aggravated apoptosis. Subsequently, shikonin upregulated autophagy and protected HUMSCs in the area surrounding contused brain tissue. Shikonin may regulate autophagy via the AMPK/mTOR signalling pathway and protect transplanted HUMSCs from apoptosis induced by hypoxia/ischemia.
\end{abstract}

Correspondence to: Professor Qichuan Zhuge or Dr Linhui Ruan, Department of Neurosurgery, The First Affiliated Hospital of Wenzhou Medical University, 123 Fanhaixi Road, Wenzhou, Zhejiang 325000, P.R. China

E-mail: zhugeqichuan@vip.163.com

E-mail: ruanlh522@163.com

*Contributed equally

Key words: shikonin, human umbilical cord mesenchymal stem cells, autophagy, apoptosis, traumatic brain injury

\section{Introduction}

Traumatic brain injury (TBI) represents a global health problem (1). The pathophysiology of brain injury following craniocerebral trauma is complex and is characterized by initial and secondary damage for several days following trauma (2). Among the numerous causes of morbidity and mortality associated with trauma, the incidence of TBI is becoming the most important cause, resulting in severe disability and mortality (3) and functional impairment, which can affect the quality of life $(4,5)$.

At present, the treatment strategies for brain trauma mainly include the control of secondary injury and promotion of neurorehabilitation through training and drug administration (6). However, this type of intervention is not enough to fully restore neural system function, so new strategies should be discovered $(1,6)$. In the last decade, some studies on stem cell transplantation as a TBI replacement therapy $(7,8)$, in animal models (9) and in the clinic have produced promising results $(10,11)$. The benefits of stem cell transplantation are multifaceted. Firstly, the ability of stem cells to undergo neural differentiation and long-distance migration to the injury site allows them to directly replace dead or dying cells $(12,13)$. Secondly, the presence of stem cells in lesions indirectly affects the microenvironment. By secreting growth factors, stem cells can promote the proliferation of nerve cells and neurotransmitter transmission $(14,15)$. However, transplanted stem cells have relatively poor survival, and their limited survival following brain injury and early death of transplanted cells limits bone marrow mesenchymal stem cell-based treatment $(16,17)$. In order to fully use the therapeutic potential of stem cells, it is crucial to determine the cause of their early death and develop strategies to improve their survival rate.

Previous studies reported that autophagy has a protective effect on mesenchymal stem cells (MSCs) under stress conditions, such as ischemia stroke $(18,19)$. Autophagy is a process of cellular self-protection that supports the homeostasis of cells under external and internal environmental stresses, including undernutrition, infection, presence of cell debris and protein aggregation $(20,21)$. Autophagy and apoptosis are the two main pathways involved in cell protection from external 
stimuli. These two processes are usually co-regulated but result in opposite cell outcomes (22). Autophagy is a self-digesting process during which a double-membrane vesicle, known as the autophagosome, is produced around the targeted organelle. Autophagosomes degrade their cargo via a lysosomal mechanism (23). The autophagy process can maintain cell fitness, nutrition, and energy levels during starvation or exposure to external stress (24). Conversely, the programmed cell death of damaged or senescent cells is known as apoptosis (24). Previous studies demonstrated that external stress stimuli may trigger one of these two processes, depending on the cellular environment $(25,26)$. The inhibition of one of these pathways can lead to the activation of the other. Triggering autophagy during programmed cell death can reduce apoptosis and prolong cell survival, whereas reducing autophagy in normal cells can increase apoptosis $(27,28)$. The regulation of autophagy may therefore provide a new mechanism to prevent cell apoptosis under stress conditions.

Shikonin is a natural naphthoquinone derivative isolated from the root of plant comfrey, and which possesses some anti-tumour, anti-inflammatory (29-31), antibacterial (32) and antithrombotic (33) activities. A study has reported that shikonin can inhibit tumour cell proliferation and metastasis by inhibiting the phosphorylation of mTOR (34). Shikonin can increase autophagy in A375 cells with 1,2 $\mu \mathrm{M}$ in $24 \mathrm{~h}$ (35). The present investigation illustrates the protective effect and underlying mechanism of shikonin in human umbilical MSCs (HUMSCs) in a hypoxic-ischemic state in vitro and in vivo.

\section{Materials and methods}

Materials. Shikonin was purchased from MedChemExpress (cat. no. HY-N0822) and was dissolved in DMSO to prepare a stock solution $\left(10 \mathrm{mg} / \mathrm{ml}\right.$; stored at $\left.-20^{\circ} \mathrm{C}\right)$ and diluted to $1.2,1.6,2,4$, and $8 \mu \mathrm{M}$ for use. 3-methyladenine (3-MA), an inhibitor of the PI3k/AMPK pathway used for creating an inhibition model of autophagy, was purchased from MedChemExpress (cat. no. HY-19312) and dissolved in double distilled water to prepare stock solutions $(5 \mathrm{mM}$ and 1,000 $\mu \mathrm{M}$ stored at $4^{\circ} \mathrm{C}$ ). DMEM and fetal bovine serum (FBS) were purchased from HyClone; GE Healthcare Life Sciences. Trypan blue stain $(0.4 \%$; cat. no. T10282) was purchased from Invitrogen; Thermo Fisher Scientific, Inc. A One-step TUNEL Apoptosis Detection kit was purchased from Beyotime Institute of Biotechnology. The purified antibodies were used as follows: Anti-AMP-activated protein kinase (AMPK; cat. no. 9158 Cell Signaling Technology, Inc.; 1:1,000); anti- $\beta$-actin (cat. no. 4967 Cell Signaling Technology, Inc.; 1:1,000); anti-GAPDH (cat. no. 5174; Cell Signaling Technology, Inc.; 1:1,000); anti-sequestosome 1 (SQSTM1)/p62 (cat. no. 5114S; Cell Signaling Technology, Inc.; 1:1,000); horseradish peroxidase-conjugated goat anti-rabbit immunoglobulin G secondary antibody (cat. no. 31460; Invitrogen; Thermo Fisher Scientific, Inc.; 1:5,000); anti-microtubule-associated protein 1A/1B-light chain 3 (LC3A/B; 1:1,000; cat. no. 12741S; Cell Signaling Technology, Inc.; 1:1,000); anti-phospho (p)-mTOR (CST; cat. no. 5536S; 1:1,000); anti-mTOR (cat. no. 2983S; Cell Signaling Technology, Inc.; 1:1,000); anti-p-AMPK (cat. no. 5759; Cell Signaling Technology, Inc.; 1:1,000); anti- $\alpha$-tubulin (cat. no. 5335; Cell Signaling Technology, Inc.; 1:1,000); anti-autophagy protein 5 (ATG5; 1:500; cat. no. ab108327; Abcam; 1:1,000); anti-Beclin 1 (cat. no. ab207612; Abcam; 1:1,000); anti-pro-caspase-3 (cat. no. ab32150; Abcam; 1:1000); anti-cleaved-caspase-3 (cat. no. ab2302; Abcam; 1:500); anti-BCL-2 (cat. no. ab182858; Abcam; 1:1,000) and anti-BAX (cat. no. ab182733; Abcam; 1:1,000). The Cell Counting Kit-8 (CCK-8), RIPA, PMSF and BCA kits were purchased from Beyotime Institute of Biotechnology.

HUMSCs. GFP-labelled HUMSCs were purchased from Cyagen Biosciences, Inc. (cat. no. HUXUC-01001). A total of $1 \times 10^{6}$ cells/vial were cryopreserved at passage 2 and stored in liquid nitrogen. These cells had been collected from the umbilical cord of healthy pregnant women with normal full-term delivery by using Wharton's Jelly. The cells were cultured (DMEM $+20 \%$ fetal bovine serum $+1 \%$ penicillin-streptomycin mixture) and placed at $37^{\circ} \mathrm{C}$ in a humidified incubator containing $5 \% \mathrm{CO}_{2}$. To stimulate hypoxic-ischemic conditions, cells were subjected to oxygen glucose deprivation (OGD).

$O G D$. HUMSCs were cultured and passaged in sugar- and serum-free medium, and the oxygen concentration in the incubator was adjusted to $0 \%$ to prepare a hypoxic environment.

Cell proliferation and treatment. Cells were seeded in a 96-well plate at a density of $10^{5}$ cells/well in $100 \mu 1$ culture medium. Cells were cultured in an incubator containing $5 \% \mathrm{CO}_{2}$ at $37^{\circ} \mathrm{C}$ for $24 \mathrm{~h}$. Subsequently, different concentrations of shikonin $(0.2,0.4,0.8,1.2$ and $1.6 \mu \mathrm{M})$ were added to the plates. Plates were incubated for 4, 8, 12 and $24 \mathrm{~h}$ under OGD pressure conditions. Subsequently, $10 \mu \mathrm{l}$ CCK- 8 solution was added per well and the plate was incubated at $37^{\circ} \mathrm{C}$ for $1 \mathrm{~h}$. A DeTie microplate reader was used to measure the absorbance at a wavelength of $450 \mathrm{~nm}$. Following CCK-8, the optimal processing time and drug concentration was obtained. All cells were divided into three groups: OGD, OGD + shikonin and OGD + shikonin + 3-MA. OGD groups were treated only with OGD. In group OGD + shikonin, shikonin was used to treat cells with OGD. In OGD + shikonin + 3-MA group, shikonin and 3-MA (5 mM for $24 \mathrm{~h}$ before OGD) were used at same time.

Animal model and tissue. A total of 18 male C57BL/6 mice (15-20 g; 9-12-week-old) were obtained from the Animal Experimental Center of Wenzhou Medical University. Mice were divided into three groups as follows: TBI, $\mathrm{TBI}+$ shikonin and TBI + shikonin + 3-MA groups $(6$ mice per group). Treatments were administered for 3 consecutive days before the TBI model was established. In mice, $50 \mathrm{mg} / \mathrm{kg}$ shikonin (36) was orally administered with 3-MA (1.5 mg/100 g), as appropriate. TBI was modelled using an Impact One ${ }^{\mathrm{TM}}$ Stereotaxic Impactor (Leica Microsystems, Inc.) at a depth of $1.0 \mathrm{~mm}$ and speed of $3 \mathrm{~m} / \mathrm{sec}$ (37) in anesthesized mice. Animal anesthesia was performed using a Reyward small animal anesthesia machine and isoflurane inhalation at 3-4\%. Once the gas completely filled the induction box $(\sim 1 \mathrm{~min})$, the animals were placed into it. Once the animals were completely anesthetized ( 2-3 min; animals 
were turned over and did not attempt resume prone position), anesthesia was maintained using $1-1.5 \%$ isoflurane, and the gas flow rate was about $300-500 \mathrm{ml} / \mathrm{min}$. GFP-HUMSCs were transplanted $24 \mathrm{~h}$ after TBI. The experimental mice were sacrificed seven days later. In each group, three mice cortical tissue surrounding the trauma was obtained and stored at $-80^{\circ} \mathrm{C}$ immediately. The other three, the brains were fixed with $4 \%$ PFA for $24 \mathrm{~h}$ with $4^{\circ} \mathrm{C}$. Then the sections were dehydrated with $30 \%$ sucrose for $48 \mathrm{~h}$ with $4^{\circ} \mathrm{C}$, embedded in optimal cutting temperature compound (OCT) and frozen, then sectioned at $10 \mu \mathrm{m}$. Animal experiments lasted a total of 11 days. All animal health, behaviour and animal welfare (including regular cleaning of cages, replacement of food and drinking water and no more than 5 mice in one cage) were monitored at the Animal Experiment Center of Wenzhou Medical University. The experimental mice were sacrificed by decapitation following an overdose of isoflurane. The study was approved by the Ethics Committee of The First Affiliated Hospital of Wenzhou Medical University (Wenzhou, China). All procedures were in compliance with the Animal Care and Use Committee of Wenzhou Medical University.

Cells transplantation. GFP-HUMSCs were trypsinized with $0.05 \%$ trypsin solution for $3 \mathrm{~min}$ at $37^{\circ} \mathrm{C}$ before transplantation. Then, HUMSCs were transferred from the culture dish to the test tube, washed with PBS for 3 times, and then injected into the TBI modelled area with a stereotaxic instrument and a micro injection needle $\left(1 \times 10^{5}\right.$ cells in $3 \mu \mathrm{l}$ at a delivery rate of $1 \mu \mathrm{l} / \mathrm{min}$.).

Trypan blue staining. Trypan blue staining was performed after shikonin treatment. HUMSCs were detached by trypsin-EDTA, and resuspended in medium. Subsequently, $200 \mu 1$ cell suspension was supplemented with $0.4 \%$ trypan blue (ratio cell suspension:trypan blue; 9:1) and incubated for $5 \mathrm{~min}$ at room temperature. Under the microscope, dead cells were stained blue, whereas living cells were transparent and colourless. ImageJ (v. 1.6.0; National Institutes of Health) was used to count the number of normal cells and dead cells in the figure. The rate of living cells was calculated by GraphPad Prism 7.0.

TUNEL immunofluorescence for HUMSC. To prepare TUNEL detection solution, TdT enzyme was added with fluorescent labelling solution at a 1:9 dilution. The HUMSCs were seeded on the glass cover in 24-well plate, and use PBS to wash redundant medium. The cell culture plate was then washed twice with PBS or Hank's Balanced Salt Solution for 10 min each time. Subsequently, $50 \mu 1$ prepared TUNEL detection solution was added to each well and incubated at $37^{\circ} \mathrm{C}$ for $1 \mathrm{~h}$ in a wet box in the dark. After staining with DAPI, a coverslip was mounted and the cells observed under a fluorescence microscope (Leica Microsystems, Inc.). The excitation wavelength range was 450-500 nm (green fluorescence) and 420-450 nm (blue fluorescence).

Western blotting (WB). Brain tissues were mashed on the ice, lysed with RIPA buffer for $30 \mathrm{~min}$ on ice and sonicated (30\% energy, $2 \mathrm{sec}$ duration, $2 \mathrm{sec}$ interval, total time $30 \mathrm{sec}$ ). Centrifuge at $12,000 \mathrm{rpm}$ for $10 \mathrm{~min}$ at $4^{\circ} \mathrm{C}$. Transfer the supernatant to a new tube. PMSF (1:100) was added, and a
BCA kit was used to determine protein concentration. Cells were washed with PBS, lysed with RIPA buffer for $30 \mathrm{~min}$ on ice and sonicated (30\% energy, 2 sec duration, 2 sec interval, total time $30 \mathrm{sec})$. PMSF (1:100) was added, and a BCA kit was used to determine protein concentration. Proteins $(60 \mu \mathrm{g})$ were separated by $10 \%$ SDS-PAGE and transferred onto PVDF membranes. The membranes were blocked $3 \mathrm{~h}(5 \% \mathrm{milk} / \mathrm{PBS}$ at room temperature). Membranes were incubated with primary antibodies against mTOR, p-mTOR, AMPK, p-AMPK, GAPDH, SQSTM1/p62, $\beta$-actin, LC3A/B, Beclin 1 ATG5 BCL2 Bax pro-caspase-3 and cleaved caspase- 3 at $4{ }^{\circ} \mathrm{C}$ overnight. Membranes were washed thrice with TBS-Tween-20 (TBS-T) and incubated with secondary antibody (dissolved in 1\% BSA; 1:5,000) at room temperature for $1 \mathrm{~h}$. Membranes were washed three times with TBS-T and exposed to Bio-Rad ChemiDoc XRS (Bio-Rad Laboratories, Inc.) under West Femto ECL Substrate (Beijing Solarbio Science \& Technology Co., Ltd.) and analysed with Image Lab 3.0 (Bio-Rad Laboratories, Inc.).

Autophagy analysis. After preparing cell protein samples, western blotting was used to verify the increase in autophagy. Autophagy is mediated by LC3BI, and increases when LC3BI is converted to LC3BII (38). Therefore, LC3BI and LC3BII, as well as the expression of their downstream products in each experimental group, were used to analyze autophagy.

Statistical analysis. The data were expressed as the means \pm standard deviation. One-way ANOVA with Dunnett's post hoc test were used to determine the statistical significance of the observed differences. Statistical analysis was performed using and GraphPad Prism 7.0 (GraphPad Software, Inc.). $\mathrm{P}<0.05$ was considered to indicate a statistically significant difference.

\section{Results}

Cell proliferation. HUMSCs were used to study the effect of shikonin (Fig. 1A) on cell proliferation. The results from the CCK-8 assay demonstrated that the optimal condition under OGD is $0.8 \mu \mathrm{M}$ shikonin treatment for the $12 \mathrm{~h}$ (Fig. 1B). In normal culture conditions, no significant cell death was observed after 24-h treatment with shikonin at different concentrations according to results from trypan blue staining. (Fig. 1C shows cell density at various concentrations under light microscope following shikonin treatment, 1D trypan blue staining and $1 \mathrm{E}$ quantification of trypan blue staining).

Shikonin protects HUMSCs from hypoxia-ischemia-induced apoptosis. Previous studies demonstrated that the maximum time for hypoxia-induced early apoptosis of mesenchymal stem cells occurs at $24 \mathrm{~h}$ (39). The present study investigated whether shikonin could reverse this process in HUMSCs. Since $12 \mathrm{~h}$ was the optimal time for drug administration, HUMSCs were exposed to OGD for $12 \mathrm{~h}$ together with $0.8 \mu \mathrm{M}$ shikonin, and apoptosis was determined using the TUNEL assay (Fig. 2D). The number of cells stained with TUNEL divided by the number of cells stained with DAPI can reflect the degree of apoptosis. Shikonin significantly inhibited TUNEL signal, and the TUNEL/DAPI ratio of the shikonin group was 
A<smiles>CC(C)=CCC(O)C1=CC(=O)c2c(O)ccc(O)c2C1=O</smiles>

C

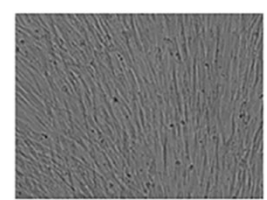

$0 \mu \mathrm{M}$

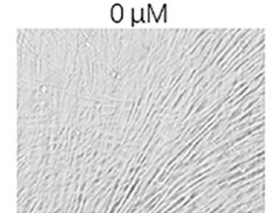

$0.4 \mu \mathrm{M}$

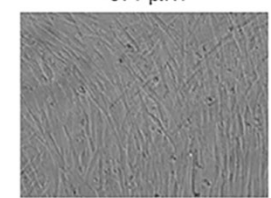

$1.2 \mu \mathrm{M}$

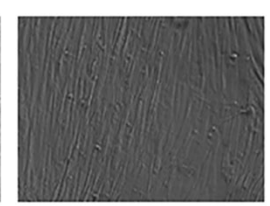

$0.2 \mu \mathrm{M}$

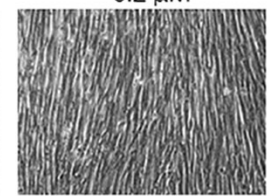

$0.8 \mu \mathrm{M}$

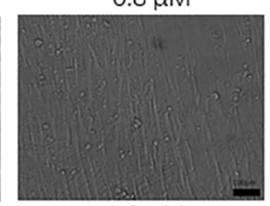

$1.6 \mu \mathrm{M}$
D

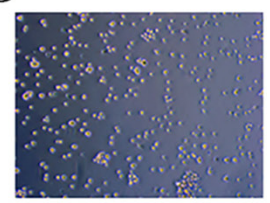

$0 \mu \mathrm{M}$

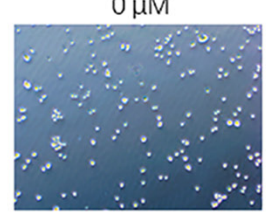

$0.4 \mu \mathrm{M}$

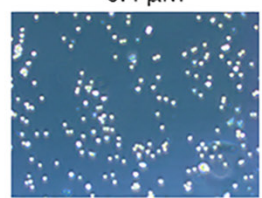

$1.2 \mu \mathrm{M}$
B

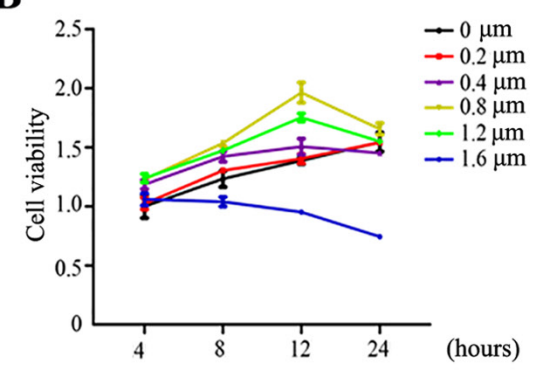

$E$

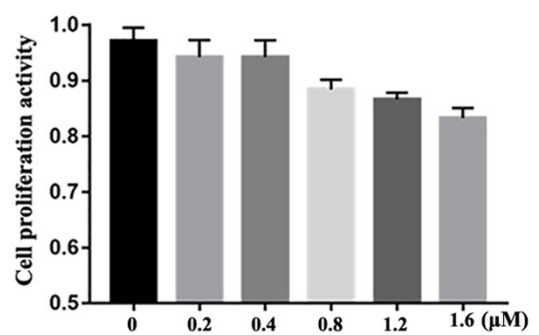

Figure 1. Toxicity and effect of shikonin on HUMSCs. (A) Chemical structure of shikonin. (B) Cell proliferation assessed with the Cell Counting Kit-8 assay. (C) HUMSCs were treated with shikonin for $24 \mathrm{~h}$ under normal conditions and were imaged with a light microscope (magnification, $\mathrm{x} 200$ ). (D) Cells under the same conditions were stained with trypan blue for 3 min and observed under a light microscope (magnification, x200). (E) Quantitative analysis of cell proliferation activity from trypan blue stainin. HUMSC, human umbilical mesenchymal stem cell.

A

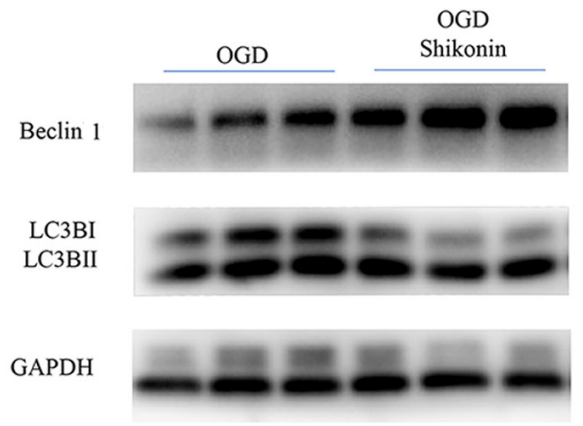

D
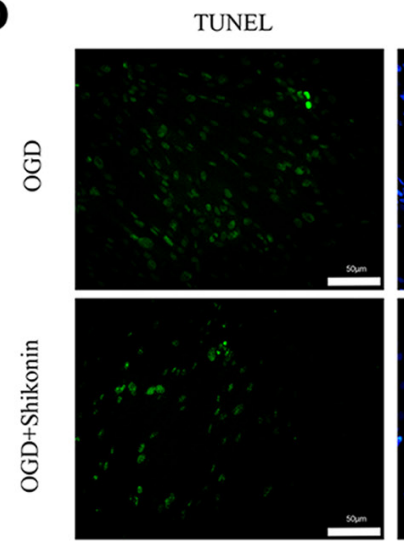

B
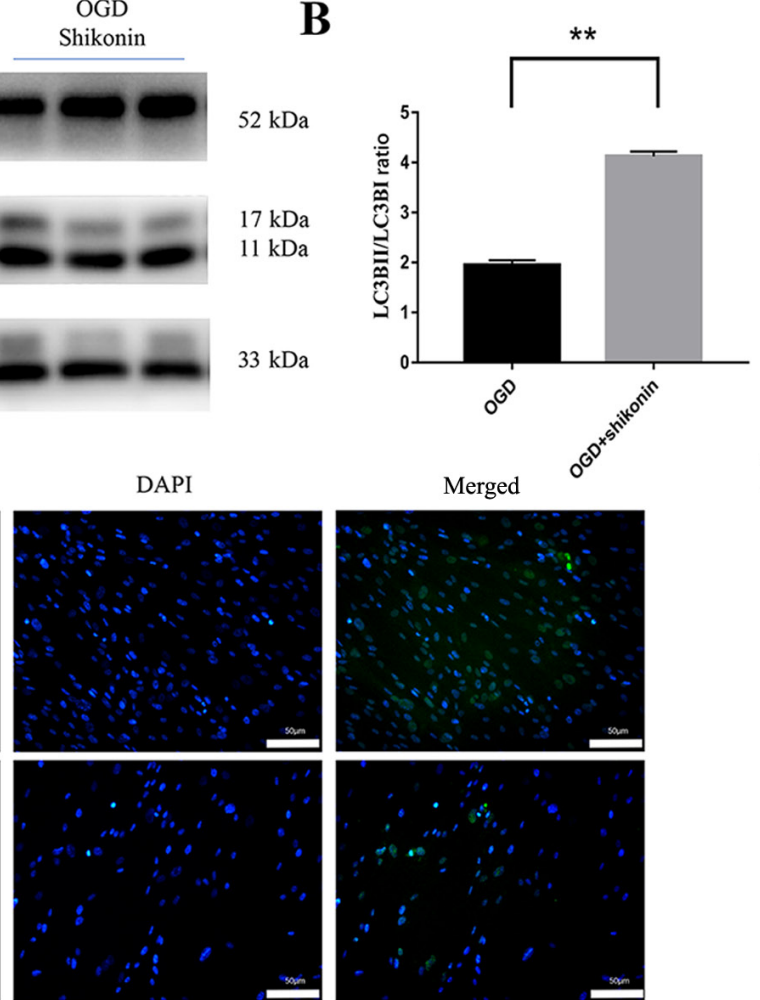
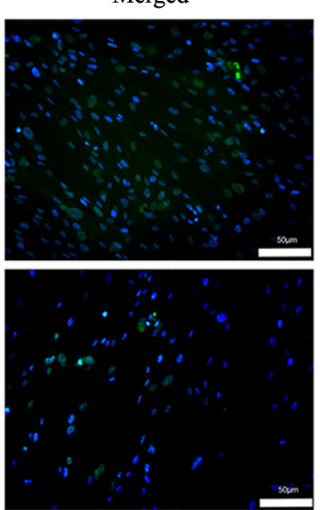

C

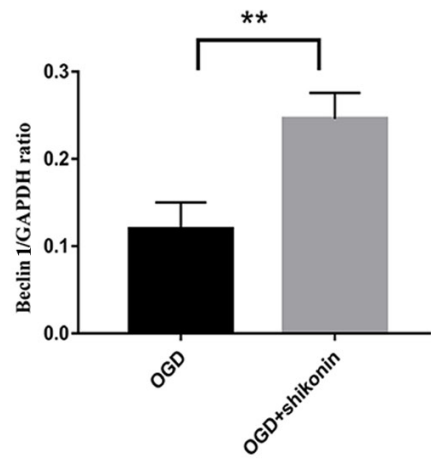

E

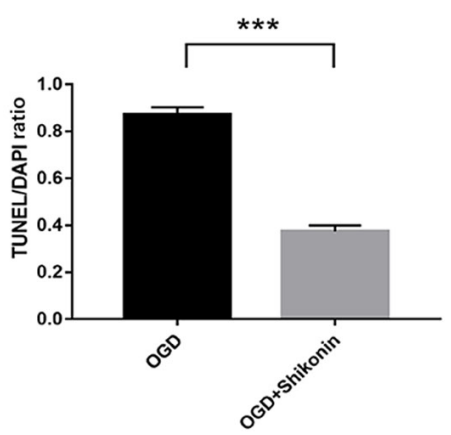

Figure 2. Shikonin reduced hypoxia-induced apoptosis and promoted autophagy in HUMSCs. Shikonin (0.8 $\mu$ M) was added to the media and cells were placed in an OGD incubator. (A) Western blotting and quantitative data of (B) LC3BII and (C) Beclin 1 in HUMSCs exposed to OGD for 12 h. (D) TUNEL staining and (E) statistical analysis of TUNEL-positive HUMSCs. Data are presented as the means \pm standard deviation. $\mathrm{n}=3$. ${ }^{* *} \mathrm{P}<0.01$ and ${ }^{* * *} \mathrm{P}<0.001$. HUMSC, human umbilical mesenchymal stem cell; OGD, oxygen glucose deprivation; LC3, microtubule-associated protein 1A/1B-light chain 3; LC3BI, cytosolic LC3; LC3BII, LC3-phosphatidylethanolamine conjugate. 

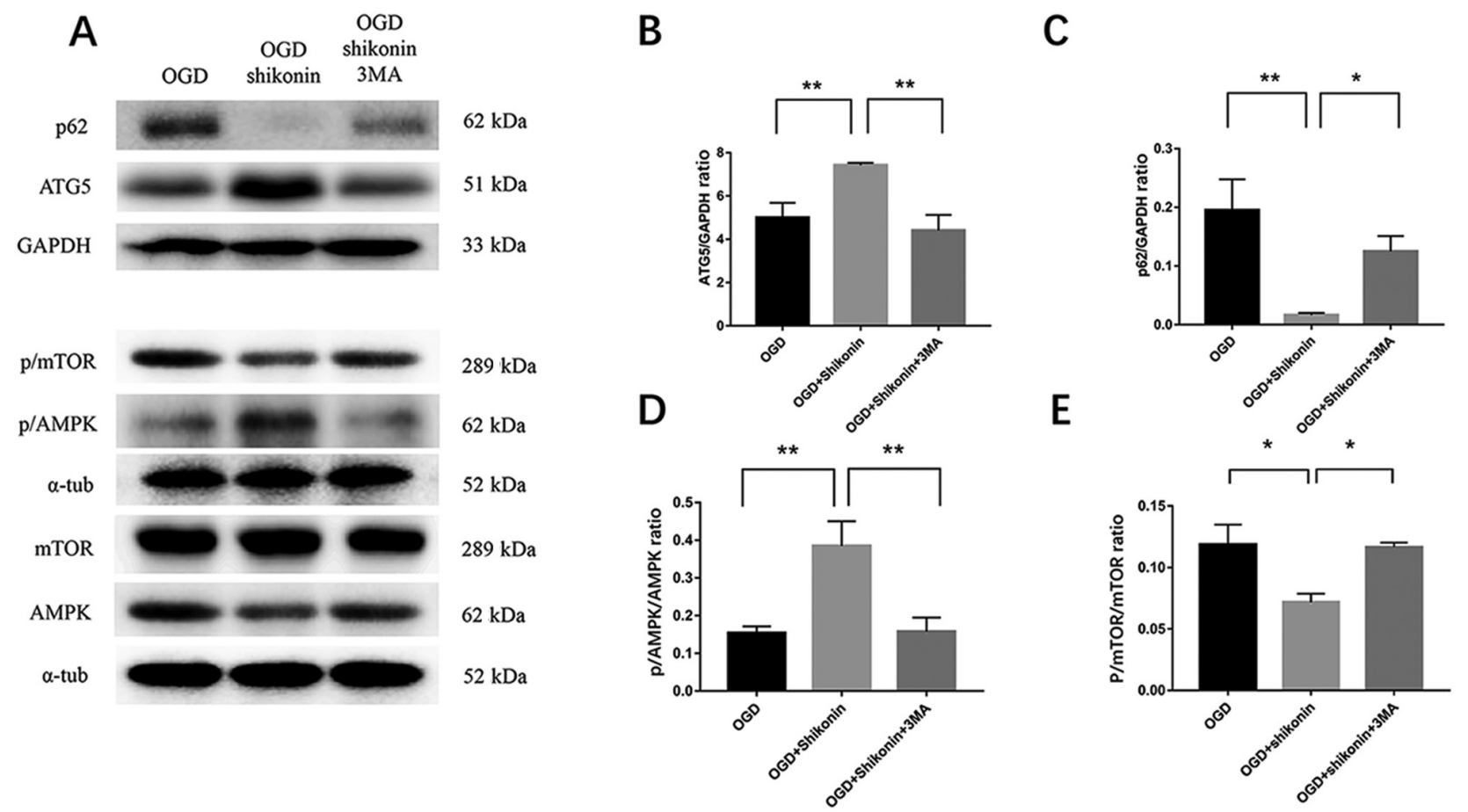

Figure 3. Shikonin enhances autophagy in HUMSCs in the hypoxic-ischemic state by regulating the AMPK/mTOR pathway. (A) Western blotting results demonstrated that (B) ATG5 and (D) p-AMPK were upregulated with shikonin treatment but downregulated after treatment with the AMPK inhibitor 3-MA. (C) p62 and (E) phosphorylation of mTOR levels were downregulated after shikonin treatment and upregulated when 3-MA was used. $n=3$. Data are presented as the means \pm standard deviation. ${ }^{*} \mathrm{P}<0.05$ and ${ }^{* *} \mathrm{P}<0.01$. HUMSC, human umbilical mesenchymal stem cell; OGD, oxygen glucose deprivation; ATG5, authophagy protein 5; AMPK, AMP-activate protein kinase; 3-MA, 3-methyladenine; P-AMPK, phosho-AMPK; P-mTOR, phosho-mTOR.

significantly lower compared with the OGD group $(\mathrm{P}<0.001$; Fig. 2E). In the present study, shikonin promoted autophagy at the concentration of $0.8 \mu \mathrm{M}$ used to decrease HUMSC apoptosis in vitro. The conversion rate of the cytosolic form of LC3 [LC3BII; ratio of LC3BII to LC3-phosphatidylethanolamine conjugate (LC3BI); $\mathrm{P}<0.01$; Fig. $2 \mathrm{~A}$ and $\mathrm{B}]$ and the expression of Beclin-1 (shikonin + OGD vs. OGD group; $\mathrm{P}<0.01$; Fig. 2A and C) were also measured. The results from western blotting demonstrated that shikonin-treated cells had significantly increased autophagy compared with OGD-treated cells.

Shikonin treatment increases autophagy and reduces apoptosis by activating the AMPK/mTOR signalling pathway. The AMPK/mTOR signalling pathway is an important regulator of autophagy in numerous cell types $(39,40)$. The present study investigated whether this signalling pathway could mediate the anti-apoptotic effect of shikonin in HUMSCs (Figs. 3 and 4). The results from western blotting demonstrated that ATG5 and p-AMPK expression was lower in the OGD group compared with the OGD + shikonin group; however, p62 and p-mTOR expression was higher in the OGD group compared with the OGD + shikonin group. Conversely, shikonin exposure resulted in a significant increase in the ratio p-AMPK/AMPK (shikonin + OGD vs. OGD group; $\mathrm{P}<0.01$; Fig. $3 \mathrm{~A}$ and D); however, the ratio $\mathrm{p}$-mTOR/mTOR was decreased (shikonin + OGD vs. OGD group; P<0.05; Fig. $3 \mathrm{~A}$ and E). Compared with the OGD group, there was a decreased expression of p62 in the shikonin group (shikonin + OGD vs. OGD group; $\mathrm{P}<0.01 ;$ Fig. $3 \mathrm{~A}$ and $\mathrm{C}$ ), and the expression of ATG5 was higher compared with the OGD group (shikonin + OGD vs. OGD group; $\mathrm{P}<0.01$; Fig. $3 \mathrm{~A}$ and $\mathrm{B}$ ). Furthermore, treatment with 3-MA lead to a decrease in p-AMPK (shikonin + OGD vs. shikonin + OGD + 3-MA group, $\mathrm{P}<0.01$ Fig. 3A and D) and p62 expression (shikonin + OGD vs. shikonin + OGD + 3-MA group; $\mathrm{P}<0.01$; Fig. 3A and C), and an increase in ATG5 (shikonin + OGD vs. shikonin + OGD + 3-MA group; P $<0.01$; Fig. $3 \mathrm{~A}$ and $\mathrm{B}$ ) and p-mTOR expression (shikonin + OGD vs. shikonin + OGD + 3-MA group; P<0.05; Fig. 3A and E). In addition, the results from TUNEL assay demonstrated that the number of TUNEL-positive cells following treatment with 3-MA and shikonin was significantly higher compared with shikonin-treated cells (shikonin + OGD vs. OGD group, $\mathrm{P}<0.01$; shikonin + OGD vs. shikonin + OGD + 3-MA group, $\mathrm{P}<0.01$; Fig. 4).

Shikonin increases the survival rate of transplanted cells in the tissues surrounding the brain contusion. HUMSCs with GFP protein gene emitting green fluorescence were found in brain tissue surrounding the TBI, suggesting that transplanted HUMSCs were able to survive and migrate to the site of injury (41). In addition, the GFP from HUMSCs was significantly increased 7 days after transplantation in the shikonin-pretreated group compared with HUMSCs alone (shikonin + TBI vs. TBI group, $\mathrm{P}<0.01$; shikonin + TBI vs shikonin + TBI + 3-MA group, $\mathrm{P}<0.05$; Fig. $5 \mathrm{~A})$. These findings suggested that shikonin may improve the number and cell viability of HUMSCs in the transplanted area.

Expression of autophagy- and apoptosis-related proteins in tissues surrounding trauma. The expression of autophagyand apoptosis-related proteins in the tissues surrounding the 
A
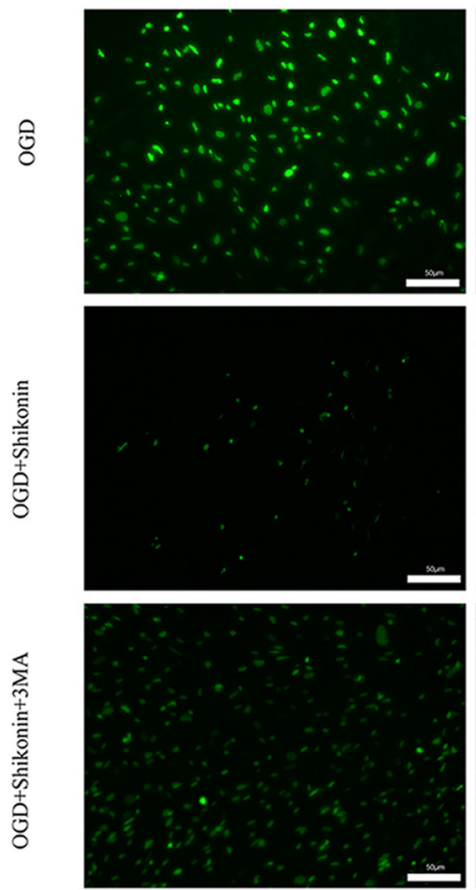

DAPI
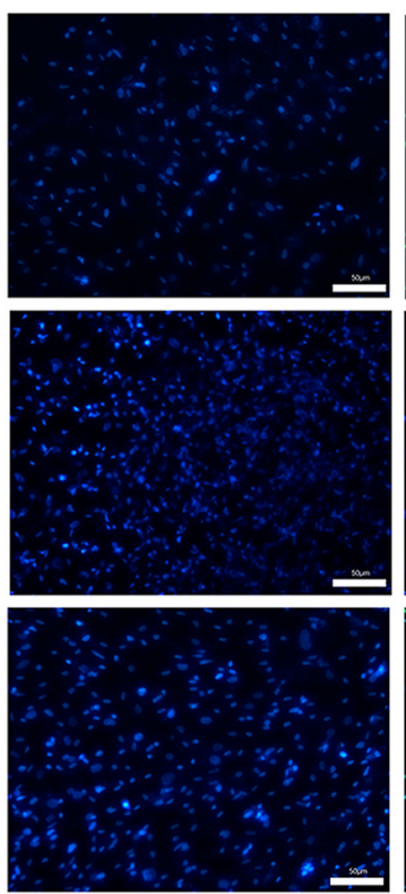

Merged
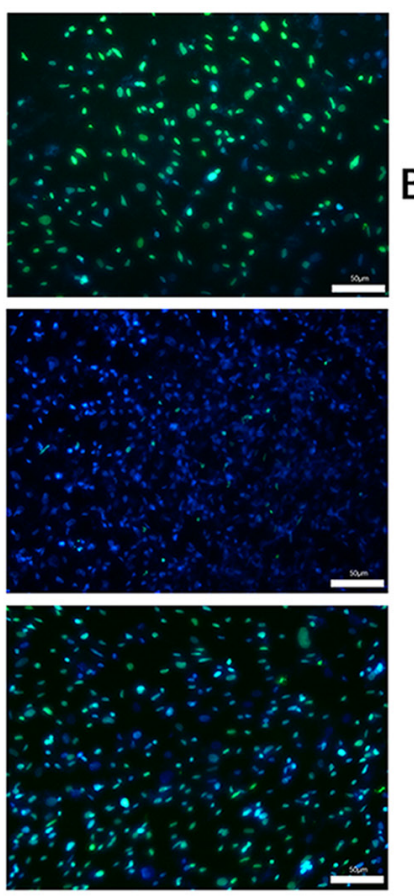

B

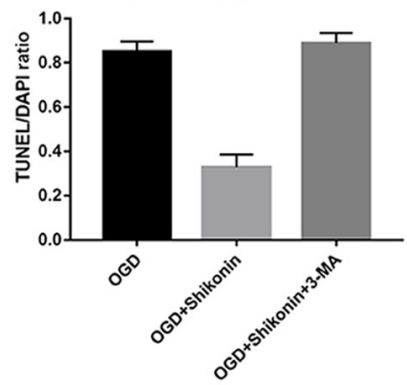

Figure 4. Shikonin enhanced human umbilical mesenchymal stem cell survival in a hypoxic-ischemic state. (A) Representative images of cells stained by TUNEL assay and counterstained with DAPI. Shikonin reduced cell death after OGD, but 3-MA reversed the effects. (B) Results from three independent experiments. ${ }^{* *} \mathrm{P}<0.01$. OGD, oxygen glucose deprivation; 3-MA, 3-methyladenine.

A

GFP
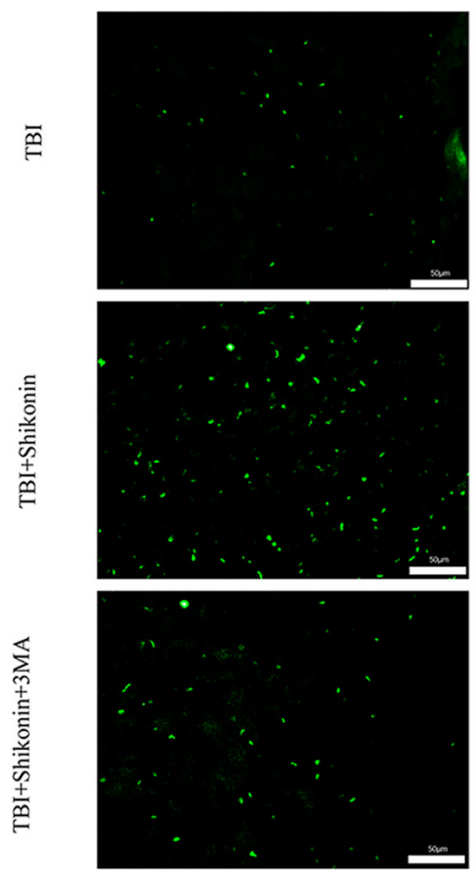

DAPI
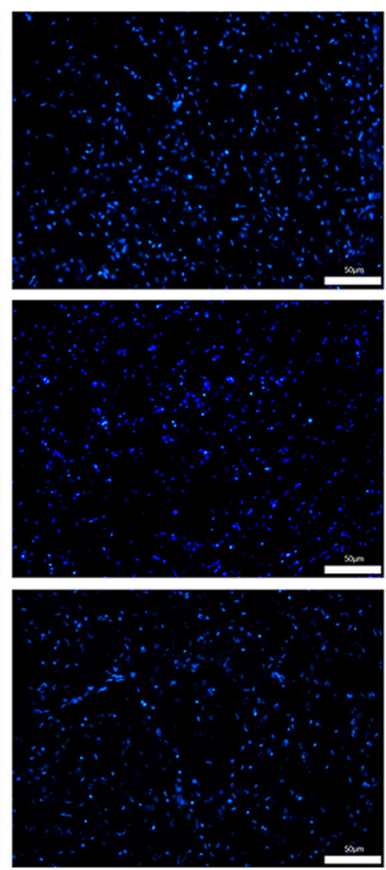

Merged
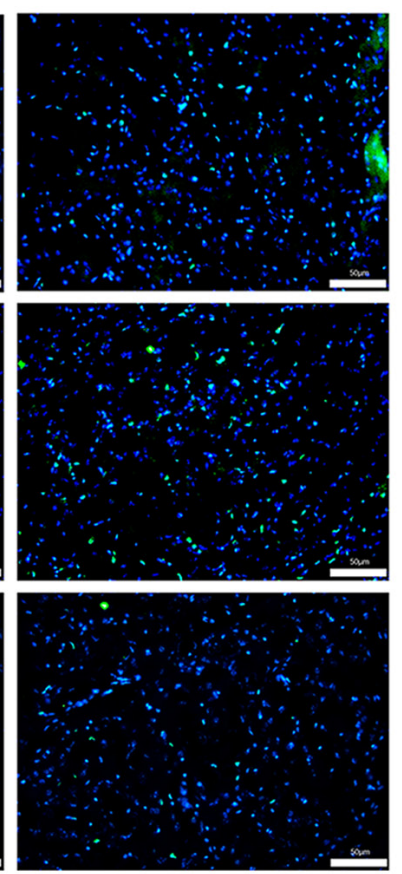

B

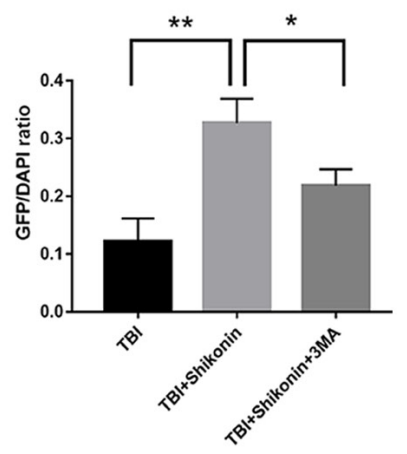

Figure 5. Shikonin increased the survival rate of transplanted cells in tissues surrounding the brain contusion. (A) Transplanted HUMSCs can survive in host tissues, as shown by transplantation of shikonin + GFP-HUMSCs, where the use of shikonin for pretreatment increased the survival rate of the transplanted HUMSCs. (B) Quantification of the number of surviving HUMSCs in shikonin-pretreated TBI mice 7 days after transplantation. $\mathrm{n}=3$. $\mathrm{P}<0.05$ and ${ }^{* *} \mathrm{P}<0.01$. HUMSC, human umbilical mesenchymal stem cell; GFP, green fluorescent protein; TBI, traumatic brain injury; 3-MA, 3-methyladenine.

brain injury $12 \mathrm{~h}$ after HUMSC transplantation was analyzed using western blotting. The results demonstrated that Beclin-1 (shikonin + TBI vs. TBI group; $\mathrm{P}<0.01$; Fig. $6 \mathrm{~A}$ and $\mathrm{B}$ ), Bcl-2/Bax ratio(shikonin + TBI vs. TBI group; $\mathrm{P}<0.01$; Fig. $6 \mathrm{H}$ ) 

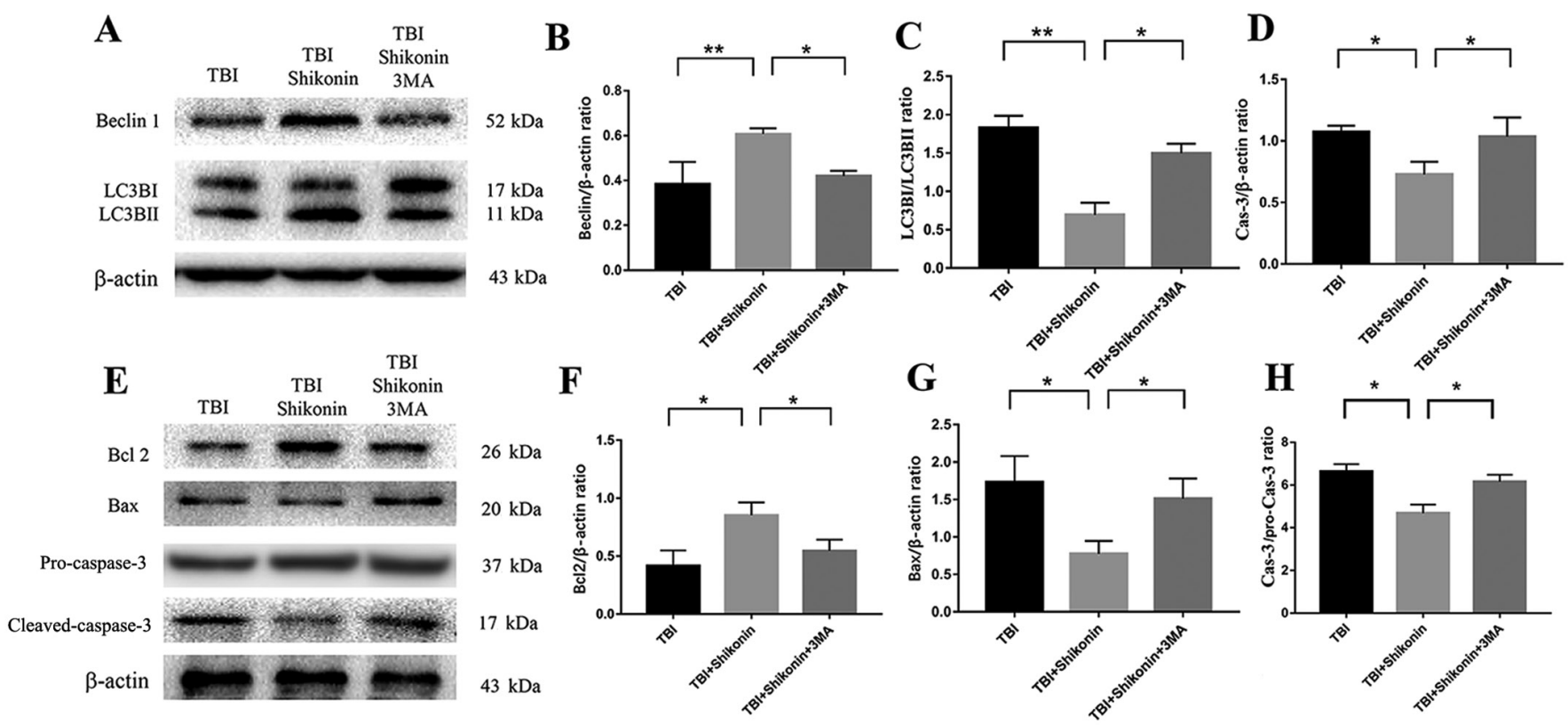

Figure 6. Transplanted stem cells reduced hypoxia-ischemia-induced apoptosis and promoted autophagy in vivo. (A) Representative western blotting of (A) autophagy- and (E) apoptosis-related proteins. (B) Beclin 1 expression and (C) LC3BII conversion was upregulated with shikonin treatment, but downregulated when 3-MA was added. (D) Caspase-3 and (G) bax expression were significantly reduced and (F) Bcl-2 expression (H) Cas-3/pro Cas-3 ratio is decreased following shikonin treatment, but reversed by addition of $3-\mathrm{MA}$. $\mathrm{n}=3$. Data are presented as the means \pm standard deviation. ${ }^{*} \mathrm{P}<0.05$ and ${ }^{* *} \mathrm{P}<0.01$. TBI, traumatic brain injury; LC3, microtubule-associated protein 1A/1B-light chain 3; LC3BI, cytosolic LC3; LC3BII, LC3-phosphatidylethanolamine conjugate; 3-MA, 3-methyladenine; cas-3, cleaved caspase-3; pro cas-3, pro caspase-3.

and Bcl-2 (shikonin + TBI vs. TBI group; $\mathrm{P}<0.05$; Fig. $6 \mathrm{E}$ and $\mathrm{F}$ ) expression, and LC3B (shikonin + TBI vs. TBI group; $\mathrm{P}<0.01$; Fig. 6A and $\mathrm{C}$ ) conversion in the shikonin + TBI group were higher compared with the TBI group. However, expression of Bax (shikonin + TBI vs. TBI group; $\mathrm{P}<0.05$; Fig. 6E and G) and caspase-3 (shikonin + TBI vs. TBI group; $\mathrm{P}<0.05$; Fig. 6E, D and $\mathrm{H}$ ) was decreased. In mice transplanted with HUMSCs and co-treated with 3-MA and shikonin, these effects were reversed (shikonin + TBI vs. shikonin + TBI + 3-MA group; $\mathrm{P}<0.05$; Fig. 6).

\section{Discussion}

Acute TBI is associated with severe complications, including tissue ischemia, excitotoxicity and overproduction of free radicals, leading to the release of inflammatory molecules, and to axonal and neuroendothelial cell damage $(42,43)$. Stem cell transplantation represents a novel treatment that could have a crucial role in the prognosis of patients with TBI (1). In the acute phase of TBI, there is severe cerebral ischemia in the region of brain contusion. At this point, the transplanted stem cells in this region are in a hypoxic and hypotrophic state, eventually resulting in severe apoptosis, which impairs the therapeutic benefits of stem cell therapy (42-44). Therefore, improving the survival rate of transplanted stem cells in this environment is crucial. In the present study, shikonin-induced autophagy served an important role in protecting mesenchymal stem cells from hypoxia-ischemia-induced apoptosis through AMPK/mTOR-related signalling pathway, which may allow MSCs to become resistant to the fluctuation of growth factors and nutrient deprivation in the ischemic microenvironment, particularly following TBI injury.
Although human and rodent brains share some physiological similarities, there are significant differences in the structure and function of their brain, which may cause rodents to respond differently to trauma (45). Furthermore, although the Glasgow coma score is an important indicator in TBI clinical experiments $(45,46)$, it is not usable in animals. Therefore, since computerized tomography, Magnetic resonance imaging and other imaging methods can be used to directly evaluate the prognosis of TBI, these methods may be able to confirm the results of the present study. Previous studies demonstrated that shikonin can improve the survival of chondrocytes, reduce chondrocyte apoptosis and promote tissue repair after spinal injury (29). These studies indicated that shikonin would be a relatively effective inhibitor of apoptosis. It has been demonstrated that shikonin can increase the expression of Beclin-1 and reduce the conversion rate of LC3-II, and the lesion volume $(35,36)$. Previous studies reported an endogenous protective mechanism of tissue ischemia and an increase in AMPK during tissue ischemia, indicating that this pathway is active during tissue ischemia (47-49). The present study demonstrated that shikonin could regulate autophagy by inhibiting the AMPK/mTOR pathway and reduce apoptosis and increase the survival rate of HMSCs, providing therefore a protective effect against contused peripheral ischemic tissue in stem cell-based therapies.

Decreased cell viability under ischemic stress is a barrier to stem cell therapy. Autophagy is a cellular response that is critical for the survival of cells under metabolic stress and energy starvation $(7,20)$. Autophagy is a catabolic process that transfers cytoplasmic components to lysosomes for their degradation (50). Hypoxia and ischemia are sources of cellular stress (51). By eliminating cellular energy supplies and 
destructing organelles damaged by free radicals, autophagy serves a vital role in cell survival $(52,53)$. Previous studies on shikonin reported that it increases autophagy $(35,54)$. In the present study, shikonin reduced the apoptosis of HUMSCs under hypoxic-ischemic conditions, which was accompanied by an increase in autophagy. Furthermore, the protective effect of shikonin was significantly decreased with the addition of the autophagy inhibitor 3-MA, which confirmed that shikonin exerted an anti-apoptotic effect by regulating autophagy in HUMSCs.

AMPK is a stress-signalling kinase that is a key regulator of energy production and depletion pathways, thus protecting cells from hypoxia and cell death $(18,40)$. One of the main mechanisms of AMPK action is the regulation of autophagy under stress conditions (48). Under hypoxia/SD or other energy shortages, AMPK may act as a sensor of cellular energy change, activated by a lower ATP/AMP ratio $(18,40)$. Furthermore, mTOR is a major downstream target of AMPK and can stimulate autophagy by inactivating the autophagy pathway inhibitor mTOR complex 1 (55). It was therefore hypothesized that the AMPK/mTOR signalling pathway could play a positive regulatory role in hypoxia, starvation and other energy stress events (55). The present study demonstrated that under hypoxic-ischemic conditions, the expression of p-AMPK was increased by shikonin, whereas the expression of p-mTOR was decreased. The opposite effects on p-AMPK and p-mTOR were observed when the AMPK inhibitor 3-MA was used together with shikonin. These results suggested that the regulation of autophagy via the AMPK/mTOR signalling pathway and, ultimately, the inhibition of apoptosis, may be considered as a potential mechanism through which shikonin inhibits apoptosis in HUMSCs.

In conclusion, shikonin promoted the survival of HUMSCs under TBI conditions. The results from the present study indicated that shikonin may regulate autophagy via the AMPK/mTOR signalling pathway and protect HUMSCs from apoptosis induced by hypoxia/ischemia. These results provided a basis for the clinical application of stem cell therapy in brain trauma treatment strategies.

\section{Acknowledgements}

Not applicable.

\section{Funding}

This work was partially supported by a grant from the Municipal Science and Technology Bureau of Wenzhou (grant no. Y20190568).

\section{Availability of data and materials}

The datasets used and/or analyzed during the current study are available from the corresponding author on reasonable request.

\section{Authors' contributions}

QZ and LR designed the study. XZ and KWu performed cell culture and TUNEL detection. XZ, LH and ZS performed the western blot analysis. KWu and JR performed CCK-8 assays and trypan blue staining. KWa and LH performed animal experiments and immunofluorescence. XZ, LH, LR and QZ performed data analysis and statistics. XZ, LH and LR confirm the authenticity of all the raw data. All authors read and approved the final manuscript.

\section{Ethics approval and consent to participate}

The present study was approved by the Ethics Committee of Wenzhou Medical University (Wenzhou, China).

\section{Patient consent for publication}

Not applicable.

\section{Competing interests}

The authors declare that they have no competing interests

\section{References}

1. Feigin VL, Nichols E, Alam T, Bannick MS, Beghi E, Blake N, Culpepper WJ, Dorsey ER, Elbaz A, Ellenbogen RG, et al; GBD 2016 Neurology Collaborators: Global, regional, and national burden of neurological disorders, 1990-2016: A systematic analysis for the Global Burden of Disease Study 2016. Lancet Neurol 18: 459-480, 2019.

2. Hu J, Chen L, Huang X, Wu K, Ding S, Wang W, Wang B, Smith C, Ren C, Ni H, et al: Calpain inhibitor MDL28170 improves the transplantation-mediated therapeutic effect of bone marrow-derived mesenchymal stem cells following traumatic brain injury. Stem Cell Res Ther 10: 96, 2019.

3. Taylor CA, Bell JM, Breiding MJ and Xu L: Traumatic Brain Injury-Related Emergency Department Visits, Hospitalizations, and Deaths - United States, 2007 and 2013. MMWR Surveill Summ 66: 1-16, 2017.

4. Bennett MH, Trytko B and Jonker B: Hyperbaric oxygen therapy for the adjunctive treatment of traumatic brain injury. Cochrane Database Syst Rev 12: CD004609, 2012.

5. Xiong Y, Mahmood A, Meng Y, Zhang Y, Zhang ZG, Morris DC and Chopp M: Neuroprotective and neurorestorative effects of thymosin $\beta 4$ treatment following experimental traumatic brain injury. Ann N Y Acad Sci 1270: 51-58, 2012.

6. Hawryluk GWJ, Rubiano AM, Totten AM, O'Reilly C, Ullman JS Bratton SL, Chesnut R, Harris OA, Kissoon N, Shutter L, et al: Guidelines for the Management of Severe Traumatic Brain Injury: 2020 Update of the Decompressive Craniectomy Recommendations. Neurosurgery 87: 427-434, 2020.

7. Mahmood A, Lu D, Yi L, Chen JL and Chopp M: Intracranial bone marrow transplantation after traumatic brain injury improving functional outcome in adult rats. J Neurosurg 94: 589-595, 2001.

8. Fairless R and Barnett SC: Olfactory ensheathing cells: Their role in central nervous system repair. Int J Biochem Cell Biol 37: 693-699, 2005.

9. Chopp M and Li Y: Treatment of neural injury with marrow stromal cells. Lancet Neurol 1: 92-100, 2002.

10. Cox CS Jr, Baumgartner JE, Harting MT, Worth LL, Walker PA, Shah SK, Ewing-Cobbs L, Hasan KM, Day MC, Lee D, et al: Autologous bone marrow mononuclear cell therapy for severe traumatic brain injury in children. Neurosurgery 68: 588-600, 2011.

11. Zhang ZX, Guan LX, Zhang K, Zhang Q and Dai LJ: A combined procedure to deliver autologous mesenchymal stromal cells to patients with traumatic brain injury. Cytotherapy 10: 134-139, 2008.

12. Skardelly M, Gaber K, Burdack S, Scheidt F, Hilbig H, Boltze J, Förschler A, Schwarz S, Schwarz J, Meixensberger J, et al: Long-term benefit of human fetal neuronal progenitor cell transplantation in a clinically adapted model after traumatic brain injury. J Neurotrauma 28: 401-414, 2011.

13. Tate MC, Shear DA, Hoffman SW, Stein DG, Archer DR and LaPlaca MC: Fibronectin promotes survival and migration of primary neural stem cells transplanted into the traumatically injured mouse brain. Cell Transplant 11: 283-295, 2002. 
14. Lee JP, Jeyakumar M, Gonzalez R, Takahashi H, Lee PJ, Baek RC, Clark D, Rose H, Fu G, Clarke J, et al: Stem cells act through multiple mechanisms to benefit mice with neurodegenerative metabolic disease. Nat Med 13: 439-447, 2007.

15. Redmond DE Jr, Bjugstad KB, Teng YD, Ourednik V, Ourednik J, Wakeman DR, Parsons XH, Gonzalez R, Blanchard BC, Kim SU, et al: Behavioral improvement in a primate Parkinson's model is associated with multiple homeostatic effects of human neural stem cells. Proc Natl Acad Sci USA 104: 12175-12180, 2007.

16. Arataki S, Tomizawa K, Moriwaki A, Nishida K, Matsushita M, Ozaki T, Kunisada T, Yoshida A, Inoue H and Matsui H: Calpain inhibitors prevent neuronal cell death and ameliorate motor disturbances after compression-induced spinal cord injury in rats. J Neurotrauma 22: 398-406, 2005

17. Fillmore N, Huqi A, Jaswal JS, Mori J, Paulin R, Haromy A, Onay-Besikci A, Ionescu L, Thébaud B, Michelakis E, et al: Effect of fatty acids on human bone marrow mesenchymal stem cell energy metabolism and survival. PLoS One 10: e0120257, 2015.

18. Xia W and Hou M: Macrophage migration inhibitory factor induces autophagy to resist hypoxia/serum deprivation-induced apoptosis via the AMP-activated protein kinase/mammalian target of rapamycin signaling pathway. Mol Med Rep 13 2619-2626, 2016.

19. Herberg S, Shi X, Johnson MH, Hamrick MW, Isales CM and Hill WD: Stromal cell-derived factor- $1 \beta$ mediates cell survival through enhancing autophagy in bone marrow-derived mesenchymal stem cells. PLoS One 8: e58207, 2013.

20. Mizushima N: Autophagy: Process and function. Genes Dev 21 2861-2873, 2007.

21. Yang Z and Klionsky DJ: Mammalian autophagy: Core molecular machinery and signaling regulation. Curr Opin Cell Biol 22: 124-131, 2010.

22. Ferraro E and Cecconi F: Autophagic and apoptotic response to stress signals in mammalian cells. Arch Biochem Biophys 462: 210-219, 2007

23. He C and Klionsky DJ: Regulation mechanisms and signaling pathways of autophagy. Annu Rev Genet 43: 67-93, 2009.

24. Danial NN and Korsmeyer SJ: Cell death: Critical control points. Cell 116: 205-219, 2004.

25. Baldwin AS: Regulation of cell death and autophagy by IKK and $\mathrm{NF}-\kappa \mathrm{B}$ : Critical mechanisms in immune function and cancer. Immunol Rev 246: 327-345, 2012

26. Palikaras K, Lionaki E and Tavernarakis N: Mitophagy: In sickness and in health. Mol Cell Oncol 3: e1056332, 2015.

27. Debnath J, Baehrecke EH and Kroemer G: Does autophagy contribute to cell death? Autophagy 1: 66-74, 2005.

28. Maiuri MC, Zalckvar E, Kimchi A and Kroemer G: Self-eating and self-killing: Crosstalk between autophagy and apoptosis. Nat Rev Mol Cell Biol 8: 741-752, 2007.

29. Bi Y, Zhu Y, Zhang M, Zhang K, Hua X, Fang Z, Zhou J, Dai W, Cui Y, Li J, et al: Effect of Shikonin on Spinal Cord Injury in Rats Via Regulation of HMGB1/TLR4/NF-кB Signaling Pathway. Cell Physiol Biochem 43: 481-491, 2017.

30. Wang L, Li Z, Zhang X, Wang S, Zhu C, Miao J, Chen L, Cui L and Qiao H: Protective effect of shikonin in experimental ischemic stroke: Attenuated TLR4, p-p38MAPK, NF- $\kappa \mathrm{B}, \mathrm{TNF}-\alpha$ and MMP-9 expression, up-regulated claudin-5 expression, ameliorated BBB permeability. Neurochem Res 39: 97-106, 2014

31. Gan L, Wang ZH, Zhang H, Zhou R, Sun C, Liu Y, Si J, Liu YY and Wang ZG: Protective effects of shikonin on brain injury induced by carbon ion beam irradiation in mice. Biomed Environ Sci 28: 148-151, 2015

32. Ding H, Jiang L, Xu J, Bai F, Zhou Y, Yuan Q, Luo J, Zen K and Yang J: Inhibiting aerobic glycolysis suppresses renal interstitial fibroblast activation and renal fibrosis. Am J Physiol Renal Physiol 313: F561-F575, 2017

33. Li F, Zhang K, Liu H, Yang T, Xiao DJ and Wang YS: The neuroprotective effect of mesenchymal stem cells is mediated through inhibition of apoptosis in hypoxic ischemic injury. World $\mathrm{J}$ Pediatr 16: 193-200, 2020.

34. Li W, Liu J and Zhao Y: PKM2 inhibitor shikonin suppresses TPA-induced mitochondrial malfunction and proliferation of skin epidermal JB6 cells. Mol Carcinog 53: 403-412, 2014.

35. Liu Y, Kang X, Niu G, He S, Zhang T, Bai Y, Li Y, Hao H, Chen C, Shou Z, et al: Shikonin induces apoptosis and prosurvival autophagy in human melanoma A375 cells via ROS-mediated ER stress and p38 pathways. Artif Cells Nanomed Biotechnol 47: 626-635, 2019.
36. Wang Z, Liu T, Gan L, Wang T, Yuan X, Zhang B, Chen $\mathrm{H}$ and Zheng Q: Shikonin protects mouse brain against cerebral ischemia/reperfusion injury through its antioxidant activity. Eur J Pharmacol 643: 211-217, 2010.

37. Romine J, Gao X and Chen J: Controlled cortical impact mode for traumatic brain injury. J Vis Exp 90: e51781, 2014.

38. New J and Thomas SM: Autophagy-dependent secretion Mechanism, factors secreted, and disease implications. Autophagy 15: 1682-1693, 2019.

39. Zhang Q, Yang YJ, Wang H, Dong QT, Wang TJ, Qian HY and $\mathrm{Xu} \mathrm{H}$ : Autophagy activation: A novel mechanism of atorvastatin to protect mesenchymal stem cells from hypoxia and serum deprivation via AMP-activated protein kinase/mammalian target of rapamycin pathway. Stem Cells Dev 21: 1321-1332, 2012.

40. Hardie DG, Ross FA and Hawley SA: AMPK: A nutrient and energy sensor that maintains energy homeostasis. Nat Rev Mol Cell Biol 13: 251-262, 2012.

41. Tang Z, Gao J, Wu J, Zeng G, Liao Y, Song Z, Liang X, Hu J, $\mathrm{Hu} \mathrm{Y}$, Liu M, et al: Human umbilical cord mesenchymal stromal cells attenuate pulmonary fibrosis via regulatory $\mathrm{T}$ cell through interaction with macrophage. Stem Cell Res Ther 12: 397, 2021.

42. Napoli I and Neumann H: Microglial clearance function in health and disease. Neuroscience 158: 1030-1038, 2009.

43. Carrico KM, Vaishnav R and Hall ED: Temporal and spatial dynamics of peroxynitrite-induced oxidative damage after spinal cord contusion injury. J Neurotrauma 26: 1369-1378, 2009.

44. Mangi AA, Noiseux N, Kong D, He H, Rezvani M, Ingwall JS and Dzau VJ: Mesenchymal stem cells modified with Akt prevent remodeling and restore performance of infarcted hearts. Nat Med 9: 1195-1201, 2003.

45. McNamara EH, Grillakis AA, Tucker LB and McCabe JT: The closed-head impact model of engineered rotational acceleration (CHIMERA) as an application for traumatic brain injury pre-clinical research: A status report. Exp Neurol 333: 113409, 2020.

46. Bolouri $\mathrm{H}$ and Zetterberg H: Animal Models for Concussion: Molecular and Cognitive Assessments - Relevance to Sport and Military Concussions. Chapter 46. In: Brain Neurotrauma. Kobeissy FH (ed). CRC Press/Taylor \& Francis, Boca Raton, FL, pp645-658, 2015.

47. Liu B, Jin J, Zhang Z, Zuo L, Jiang M and Xie C: Shikonin exerts antitumor activity by causing mitochondrial dysfunction in hepatocellular carcinoma through PKM2-AMPK-PGC1 $\alpha$ signaling pathway. Biochem Cell Biol 97: 397-405, 2019.

48. Jiang S, Li T, Ji T, Yi W, Yang Z, Wang S, Yang Y and Gu C: AMPK: Potential Therapeutic Target for Ischemic Stroke. Theranostics 8: 4535-4551, 2018.

49. Qiang L, Wu C, Ming M, Viollet B and He YY: Autophagy controls p38 activation to promote cell survival under genotoxic stress. J Biol Chem 288: 1603-1611, 2013.

50. Zhang H, Bosch-Marce M, Shimoda LA, Tan YS, Baek JH, Wesley JB, Gonzalez FJ and Semenza GL: Mitochondrial autophagy is an HIF-1-dependent adaptive metabolic response to hypoxia. J Biol Chem 283: 10892-10903, 2008.

51. Bachmann J, Ehlert E, Becker M, Otto C, Radeloff K, Blunk T and Bauer-Kreisel P: Ischemia-Like Stress Conditions Stimulate Trophic Activities of Adipose-Derived Stromal/Stem Cells. Cells 9: 1935-1955, 2020.

52. Hamacher-Brady A, Brady NR, Logue SE, Sayen MR, Jinno M, Kirshenbaum LA, Gottlieb RA and Gustafsson AB: Response to myocardial ischemia/reperfusion injury involves Bnip3 and autophagy. Cell Death Differ 14: 146-157, 2007.

53. Gwon SY, Ahn J, Jung CH, Moon B and Ha T-Y: Shikonin Attenuates Hepatic Steatosis by Enhancing Beta Oxidation and Energy Expenditure via AMPK Activation. Nutrients 12: $1133-1145,2020$

54. Wang F, Mayca Pozo F, Tian D, Geng X, Yao X, Zhang Y and Tang J: Shikonin Inhibits Cancer Through P21 Upregulation and Apoptosis Induction. Front Pharmacol 11: 861-873, 2020.

55. Kapahi P, Chen D, Rogers AN, Katewa SD, Li PW, Thomas EL and Kockel L: With TOR, less is more: A key role for the conserved nutrient-sensing TOR pathway in aging. Cell Metab 11: 453-465, 2010.

This work is licensed under a Creative Commons Attribution-NonCommercial-NoDerivatives 4.0 International (CC BY-NC-ND 4.0) License. 